\title{
Challenges for Digital Product and Process Design Systems at BMW
}

\author{
Dietmar Trippner, Stefan Rude, and Andreas Schreiber \\ \{dietmar.trippner, stefan.rude, andreas.as.schreiber\}@bmw.de
}

\begin{abstract}
The methods of model based product development are today well understood by industry across branches as in automotive and aerospace including their supply chain. Actual challenges of these industries however let question the capacity of the concept. Such major changes in Automotive are light weight design, electro-mobility as well as modern mobility concepts, all increasing the product complexity. Increasingly an overall approach is requested that is able to consistently integrate Requirements, Functions, Logic and Physics (RFLP approach). Not only with regards to mechanical aspects, but also considering demands of electric/electronic as well as software development.

The Systems Engineering approach is addressing the consistent availability and linkage of product information. Well established in aerospace and defence since decades the concept arrives in Automotives just right now, driven for example by the introduction of integrated product development environments. However the Systems Engineering introduction to an automotive OEM still might be a challenge. Typical obstacles are different structured and detailed design requirements (customer requirements versus vehicle requirements and component characteristics) as well as missing consideration of configuration, effectivity and maturity. Additionally the two dimensions of automotive consistency (Vehicle to component - vertical integration - versus integration of early concept stages via development, verification, approval until the final start of production - horizontal integration) contribute to the challenge.

Finally also an improvement of interdisciplinary interaction is needed (mechanics including simulation, electric/electronics and software). The introduction of Systems Engineering is not only challenging the coordination of process IT infrastructure (Authoring systems, TDM and PDM) but also needs to consider organizational aspects (process and appropriate organizational units, integration of design partners and suppliers). Frequent acquisitions among IT system vendors - especially in the $\mathrm{CAD} / \mathrm{PLM} / \mathrm{CAE}$ market - as well as the need to select a system considering functional as well as economic aspects drive the demand for open interfaces.

The presentation highlights the success and payoff of the application of Systems Engineering for Automotive processes. Implications for the process IT infrastructure coordination will be introduced.

Necessary process IT adaptations will be addressed using target templates for processes, design system infrastructure, integration and mapping.

Finally BMW's concept to enforce openness from all OEM, Supplier and System Vendor based on the Codex of PLM Openness (CPO) will be discussed. Success stories as well as lessons learned will be shared.
\end{abstract}

Keywords: Systems Engineering, PDM, TDM, CAE, Simulation, RFLP, Codex of PLM Openness (CPO) 


\section{Introduction}

Digital Product- and Process development are getting more and more important at BMW and all related brands, as Mini or Rolls-Royce, for example. See Fig. 1. and Fig.2.

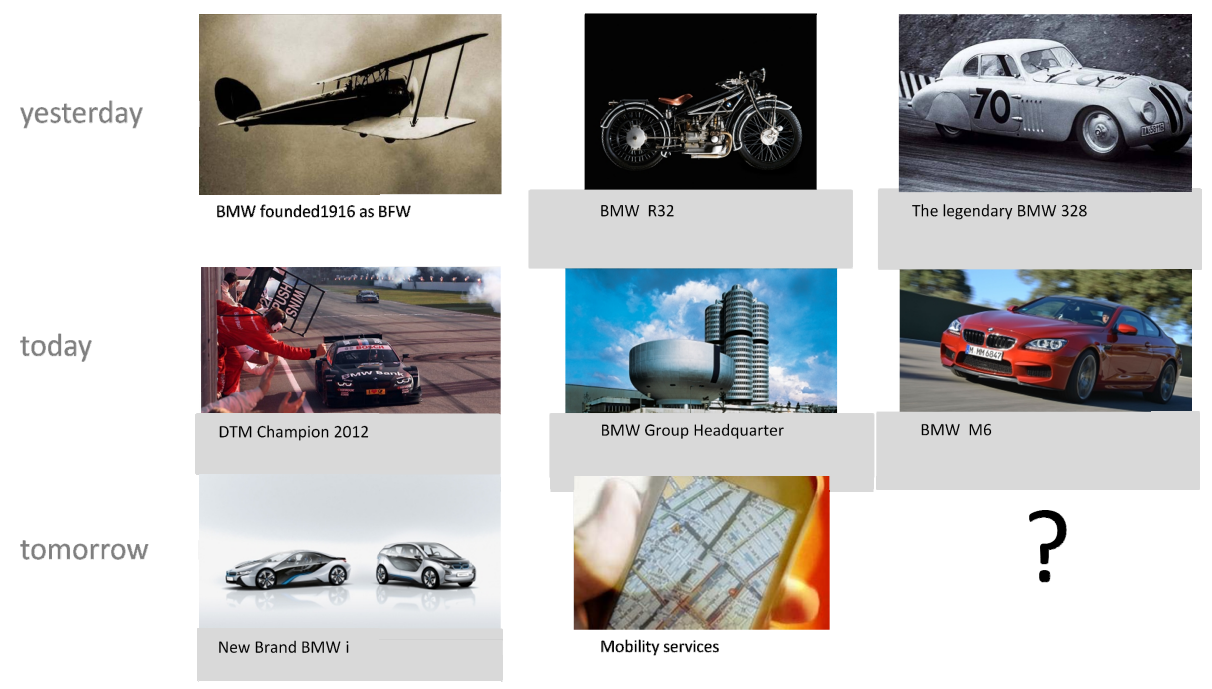

Fig. 1. BMW Group - Company Portrait

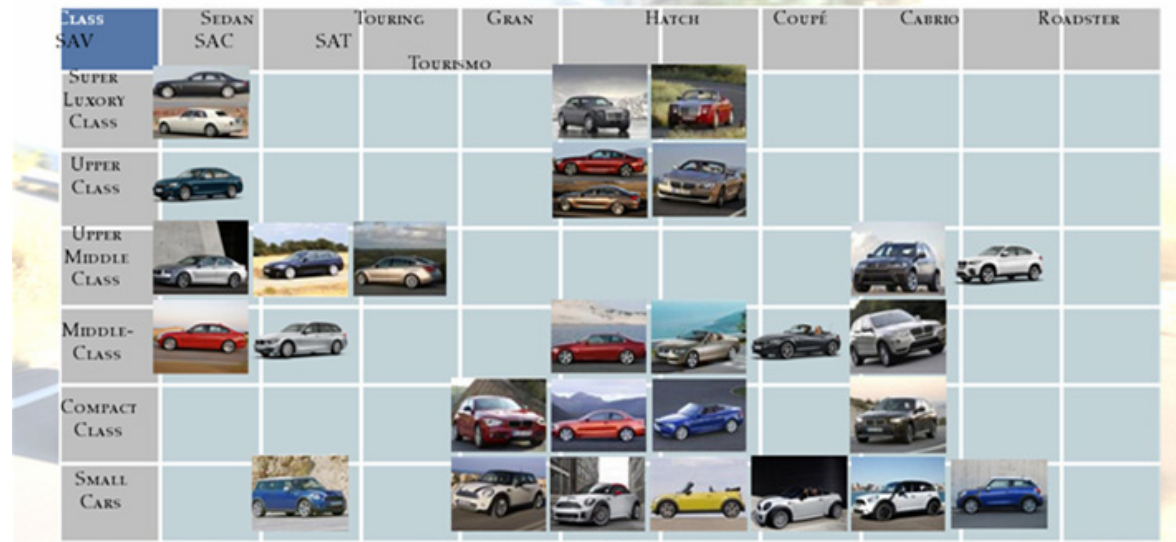

Fig. 2. The BMW Group Product Portfolio

Major challenges in Automotive are light weight design, electro-mobility as well as modern mobility concepts, all increasing the product complexity (see Fig. 3). 


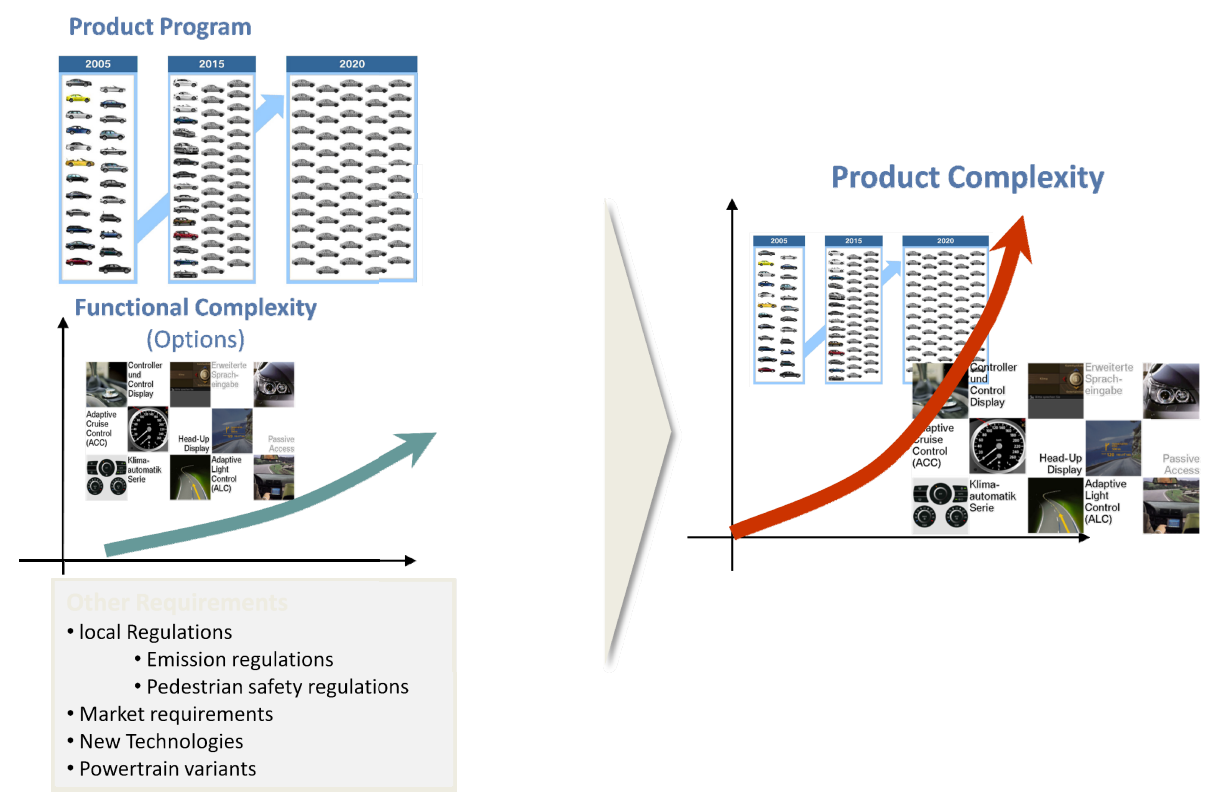

Fig. 3. Increased product complexity in product design

\section{Requirements, Coming from the Development Process}

To meet these different requirements for the development of a complex product program a highly optimized design methodology is needed (see Fig. 4).

The Systems Engineering V-Model is a good base for such a design process (see Fig. 5).

The use of virtualization dramatically increased over the years. But its success was created by introducing many IT applications covering the various aspects of simulation. The next level of efficiency therefore needs to address the topic of consistent integration of all these applications (see Fig. 6). 


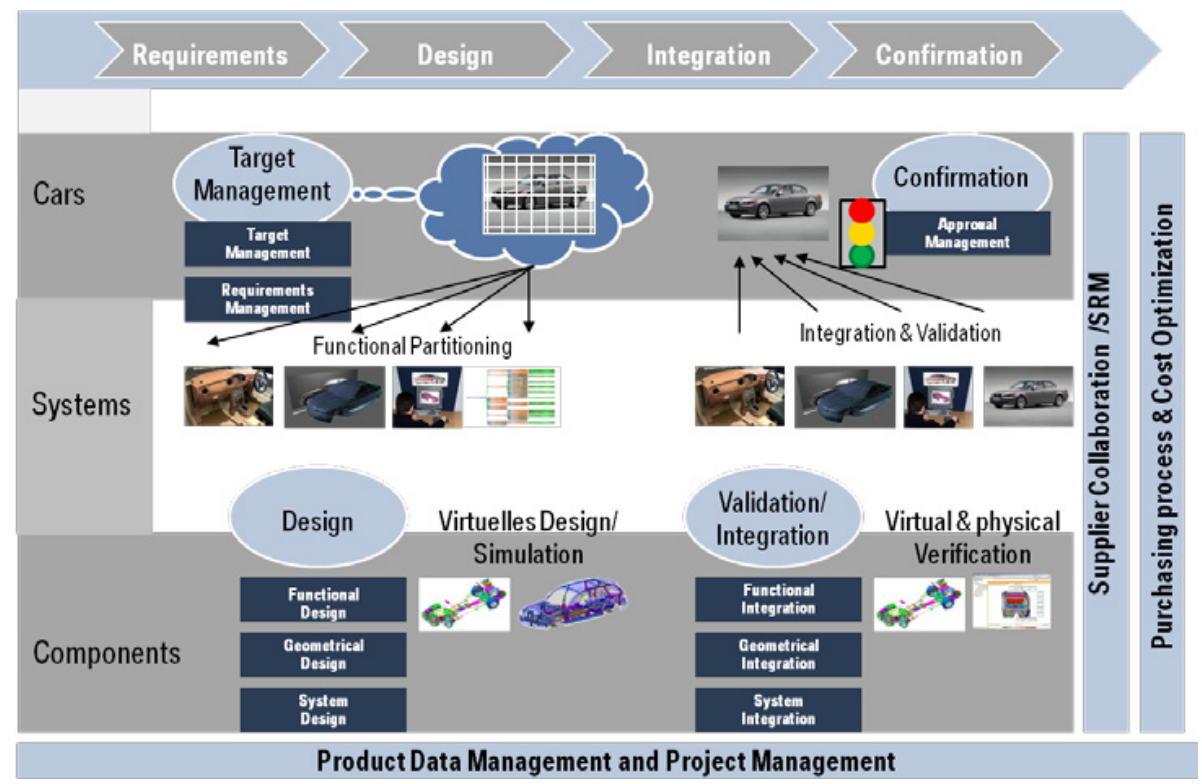

Fig. 4. Design process principles

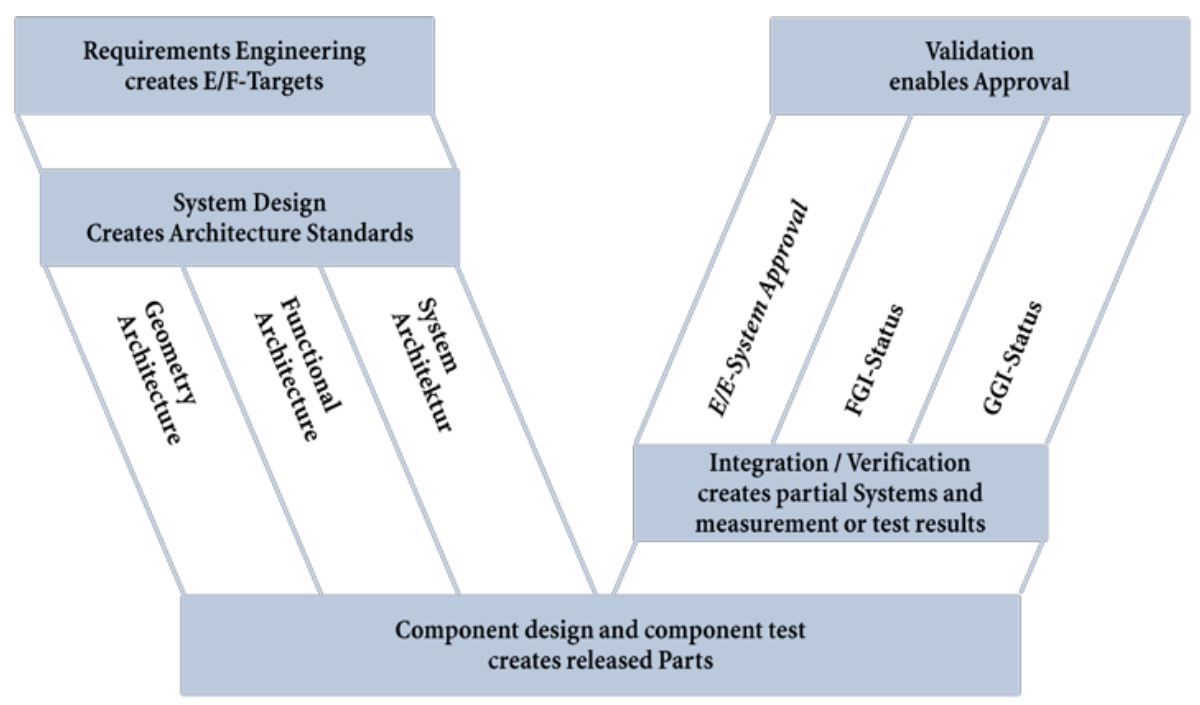

Legend: E/F - Properties/ Functions, E/E - Electric/ Electronic, FGI - Functional Design and Integration, GGI - Geometrical Design and Integration

Fig. 5. The development process in the V-Model 


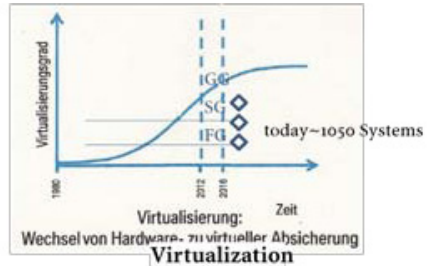

\section{Challenge:}

Consistent integrated Solutions

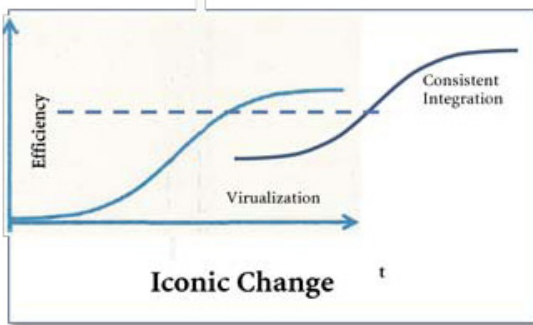

Business:

- Standardized user concept for different applications

- Compatibility of functionalities in different applications

- Re-use of data from previous processes

IT:

- Manage IT-System landscape Complexity

Manage Operations Challenge - Ensure Data Consistency and Actuality

Fig. 6. Product design challenges 